\title{
Generalization and false memory in acquired equivalence
}

\author{
Maria Alejandra de Araujo Sanchez \& Dagmar Zeithamova \\ University of Oregon
}

Corresponding author:

\author{
Dagmar Zeithamova \\ Department of Psychology \\ 1227 University of Oregon \\ Eugene, OR \\ 97403 \\ dasa@uoregon.edu \\ $541-346-6731$
}

Word count: 10,490 (including citations) 


\begin{abstract}
Memory allows us to remember specific events but also combine information across events to infer new information. New inferences are thought to stem from integrating memories of related events during encoding but can be also generated on-demand, based on separate memories of individual events. Integrative encoding has been argued as dominant in the acquired equivalence paradigm, where people have a tendency to assume that when two faces share one preference, they also share another. A downside may be a loss of source memory, where inferred preferences are mistaken for observed ones. Here, we tested these predictions of the integrative encoding hypothesis across five datasets collected using variations of the acquired equivalence paradigm. Results showed a statistically reliable but numerically small tendency to generalize preferences across faces, with stronger evidence for on-demand inferences at retrieval rather than spontaneous integration during encoding. A newly included explicit source memory test showed that participants differentiated learned from inferred preferences to a high degree, irrespective of whether they generalized preferences across faces. False memory was however increased in participants who made generalization decisions faster, as would be predicted by integrative encoding. The results indicate that generalization in acquired equivalence may result from integrated representations that facilitate new inferences at the expense of source memory, as proposed previously. However, they also demonstrate that on-demand retrieval-based processes may play a larger role in this paradigm than previously thought. Finally, the results indicate that reaction times may be more sensitive than performance as a means to assess representations underlying behavior. More broadly, the study informs current theories of generalization and knowledge representation and provides new insights into how memory biases decisions.
\end{abstract}

Keywords: Memory integration, generalization, acquired equivalence, integrative encoding 
Memory allows us to remember specific details of individual events but also connect information across events to form general knowledge and answer novel questions. Memory for specific events is well known to rely on the episodic memory system and the hippocampus (Diana et al., 2007; Eichenbaum, 2001; Scoville \& Milner, 1957; Squire, 1992). Interestingly, many forms of generalization also involve the hippocampus (Mack et al., 2018; Schlichting \& Preston, 2017; Zeithamova \& Bowman, 2020). Current theories argue whether hippocampalbased generalization is fully explainable as a recursive retrieval of specific memories or whether it includes a unique memory integration process that leads to the formation of generalized representations combining elements across experiences (Kumaran \& McClelland, 2012a; Zeithamova, Schlichting, et al., 2012; Zeithamova \& Bowman, 2020). Because generalization judgments can be based on either type of representation - integrated representations or on-demand inference from separate memories - identifying the representations underlying behavior can be a challenge. As one means, prior work suggests that generalization judgments based on integrated representations tend to be faster and more accurate than those based on demand-driven inference from separate representations (Schlichting et al., 2014; Shohamy \& Wagner, 2008). Furthermore, it was suggested that integration of related memories may lead to false memory for the inferred links between memories, although empirical evidence is limited (Carpenter \& Schacter, 2017, 2018a; Shohamy \& Wagner, 2008; Varga et al., 2019). Some instead found that generalization is accompanied by better rather than worse source memory (Banino et al., 2016), arguing that both depend on the quality of specific memories of individual events. Thus, the question of how humans represent and integrate related experiences in service of generalization remains open.

Acquired equivalence is an established paradigm focusing on spontaneous generalization, shown to involve the hippocampus in both humans and animal models (Doll et al., 2015; Edwards et al., 1982; Honey \& Hall, 1991; Meeter et al., 2009; Shohamy \& Wagner, 2008). In this paradigm, two stimuli-we will call them pairmates-share the same associate or require the same response. The tendency to generalize a piece of information from one pairmate to the other is tested. For example, participants learn through feedback-based training that two stimuli, such as Face 1 and Face 2, share a common preference, such as both preferring Scene 1 over Scene 1'. In addition, they learn that Face 1 also prefers Scene 2 over Scene 2'. Subsequent test includes a critical untrained preference, such as whether Face 2 also prefers Scene 2 over Scene 2' (Figure 1). Response bias - choosing Scene 2 over Scene 2 ' reliably more often than $50 \%$-is then taken as evidence for generalization, or "acquired equivalence", across the two 
faces. Generalizing knowledge between pairmates requires participants to link information across memories acquired at different times.
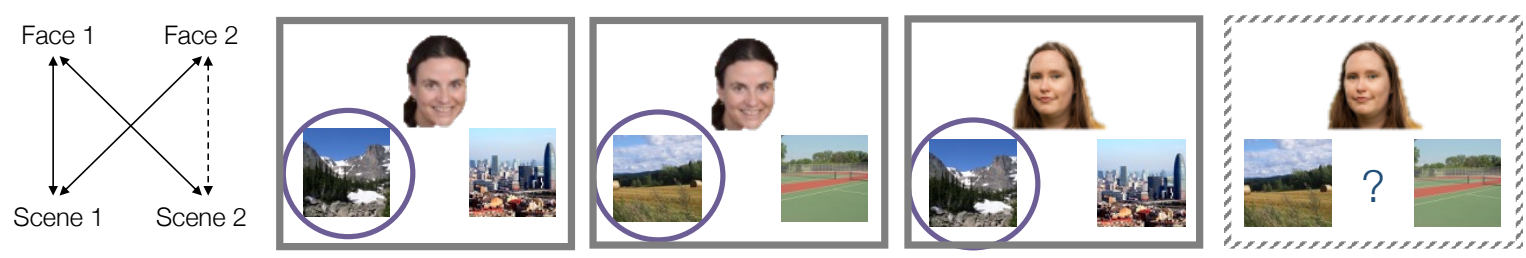

Figure 1. Stimulus structure in the acquired equivalence paradigm. Participants learn scene preferences for a set of faces via feedback-based learning. Correct preference for each face is highlighted in this figure by a circle (Scene 1 and Scene 2), the other scene is a foil scene not preferred by that face (Scene 1' and Scene 2'). Two faces (Face 1 and Face 2) are pairmates and share a preference for Scene 1 (here, mountain over city). A second preference for Face 1 is also trained (here, fields over tennis court). Test includes trained associations and the untrained Face2-Scene2 associations (dashed line, dashed border). Response bias (here, the tendency to select fields over tennis court as a preference for Face 2) would indicate spontaneous generalization of preferences across faces. Participants are not informed about the structure of the stimuli and they are not told that the test includes preferences they had not studied.

Whether generalization in acquired equivalence is based on integration of related associations during encoding (Shohamy \& Wagner, 2008) or whether it relies on recursive retrieval of individually learned associations (Kumaran \& McClelland, 2012b) has been debated. A strong argument for integration during encoding came from a neuroimaging study by Shohamy and Wagner (2008). As neural evidence, individual differences in generalization were predicted by hippocampal activation at encoding rather than retrieval. However, a striking pattern of behavioral data provided just as compelling evidence. Participants showed a strong response bias on untrained trials, generalizing preferences across pairmate faces on about $80 \%$ of trials, although subsequent studies found somewhat lower generalization rates (Doll et al., 2015; Duncan et al., 2012). There were, however, also large individual differences in the tendency to generalize, which were inversely correlated with reaction time cost for untrained trials. Specifically, reaction times were slower on untrained than trained trials overall, but the differences were minimized in participants who showed a strong tendency to generalize. Based on these findings, Shohamy and Wagner (2008) argued that generalization does not occur through recombination of separate memories at retrieval (which would require extra processing and longer reaction times on untrained trials) but rather was a result of memory integration at encoding. Presumably, people reactivate prior related memories during encoding, forming representations that link elements across related events as if they were directly experienced. 
A possible trade-off of integration may be a loss of source memory (Carpenter \& Schacter, 2017; Shohamy \& Wagner, 2008; Varga et al., 2019). As anecdotal evidence, Shohamy \& Wagner (2008) conducted an informal debriefing during which only two participants spontaneously reported having noticed that they were tested on associations they had not studied, and both of them had low generalization scores. Presumably, most participants had formed integrated memories that already represented inferred links between all elements (e.g., F1/F2/S1/S2 memory), and thus did not notice that the untrained relationships were novel. However, as false memory for the untrained association was not tested formally, the evidence for mistaking inferred associations for observed ones remains anecdotal. The goal of the current study was to address this gap, behaviorally replicating the study by Shohamy and Wagner (2008) with an addition of a source memory task to explicitly measure false memory for the untrained associations. Based on the memory integration hypothesis, we predicted that participants will tend to generalize preferences between face pairmates, accompanied by minimal reaction time costs and a high degree of false memory for the untrained preferences.

An alternative to integrative encoding of related memories is their encoding as separate representations. Single-system accounts of generalization point out that one does not need to assume the existence of generalized memories that integrate experiences (Nosofsky, 1986; Kumaran, 2012). Even when related events are initially encoded as separate memories, one may still infer novel untrained relationships on-demand through a recursive retrieval of the individual associations (Kumaran, 2012; Kumaran \& McClelland, 2012b). Several studies reported unique hippocampal activation on generalization test trials, consistent with retrieval-based accounts (Preston et al., 2004; Schlichting et al., 2014; Zalesak \& Heckers, 2009; Zeithamova \& Preston, 2010). However, such retrievalbased inference is thought to result in lower generalization rates and increased response time compared to integration-based generalization (Schlichting et al., 2014; Zeithamova \& Preston, 2017).

The role of retrieval-based inference in acquired equivalence is unclear as few studies besides Shohamy \& Wagner (2008) reported data that could clearly differentiate between integrative encoding vs. retrieval-based process. However, additional insights are provided by work on memory integration using other relational generalization paradigms, most prominently the associative inference paradigm (Bunsey \& Eichenbaum, 1996; Preston et al., 2004). In associative inference, participants first learn overlapping paired associates on separate trials (A-B, B-C; e.g. pear-car, car-watch) and then are tested on inferred associations ( $\mathrm{A}-\mathrm{C}$; pear-watch). Participants are informed (sometimes before 
encoding, but always at least before the test) about the structure of the stimuli and are instructed that there is a correct choice on the inference trial, such that they should pick $C$ when probed with $A$ due to the common associate, $B$. Although the explicit instruction differentiates associative inference paradigm from the more incidental generalization in acquired equivalence, both paradigms are thought to involve common processes, most importantly memory integration that involves linking of information across experiences and the hippocampus (Schlichting \& Preston, 2017; Zeithamova, Schlichting, et al., 2012; Zeithamova \& Bowman, 2020).

The work on associative inference has provided some evidence consistent with the integrative encoding hypothesis as well as some evidence highlighting retrieval-based strategies. Using brain-decoding approaches (Norman et al., 2006; Polyn et al., 2005), associative inference studies were able to test and confirm the prediction from Shohamy and Wagner (2008) that prior memories are reactivated during encoding of overlapping events, and that reactivation leads to memory integration, supporting inference across events (Zeithamova, Dominick, et al., 2012; Zeithamova \& Preston, 2017). Importantly, associative inference studies also indicated that related events do not have to be integrated and may remain separated in memory, influenced for example by temporal proximity, reward, strength of pre-existing memories or explicit instruction (Barron et al., 2020; Richter et al., 2016; Schlichting et al., 2015; Tse et al., 2007; Zeithamova \& Preston, 2017). Separate memories can still be used to generate inferences ondemand, albeit such inferences tend to be slower and less accurate (Schlichting et al., 2014).

Studies on associative inference also aimed to explicitly address the question to what degree memory integration relates to a possible loss of episodic details, such as mistaking inferred associations for observed ones. Carpenter and Schacter $(2017,2018 a)$ tested the idea that false memory and generalization may be flip sides of the same coin. As in other associative inference paradigms, participants encoded pairs of overlapping events (man-toy truck, toy truck-boy) and were then tested on inferential relationships requiring linking information across the two episodes (man-boy). A novel manipulation was presentation of peripheral details (such as background objects or monetary values) that differed between overlapping events. Participants showed an increased incidence of false memories for details surrounding the related events, such as falsely attributing a brown couch to the boy although it was seen with the man instead. False memories specifically increased after a successful inference across events, indicating that linking the two events in response to a test question created an integrated memory representation, but sacrificed the information about the 
source of individual details. A lack of false memory after encoding but before the inference test indicated that integration happened on-demand during retrieval rather than spontaneously during encoding. Interestingly, older adults tested in the same paradigm did not show an increased incidence of false memories for peripheral details after inference (Carpenter \& Schacter, 2018b), indicating that the trade-off does not always happen.

Banino and colleagues (2016) aimed to address the same question using explicit source memory probes. After each test trial, participants were asked whether the trial was direct (directly learned $A B, B C$ trials) or indirect (i.e., testing the $A C$ inference). Source memory for $A B$ and $B C$ trials positively predicted $A C$ inference success, with the retrieval-based model of generalization REMERGE well accounting for the data. The authors concluded that episodic memory accuracy and generalization go hand in hand rather than trade-off. However, some false memory for AC trials occurred, as participants' source memory judgments on $A C$ trials did not exceed $70 \%$ (chance $=50 \%$ ). Furthermore, as the relationship between $A C$ inference success and $A C$ source memory was not reported, this study did not directly address the question whether linking information across episodes relates to false memories for inferred information, leaving the critical prediction of the integrative encoding hypothesis untested.

Jointly, the body of behavioral and neuroimaging studies indicate an important role for both integration during encoding and on-demand retrievalbased processes in associative inference. In contrast, the initial study by Shohamy and Wagner (2008) indicated that integration during encoding is the dominant mechanism in the more incidental acquired equivalence paradigm, although evidence from subsequent acquired equivalence studies was less clearcut (Doll et al., 2015; Duncan et al., 2012; Foerde \& Steinglass, 2017). No study yet explicitly tested the anecdotal evidence of false memory for untrained preferences in this paradigm. The goal of the current study was to address this gap and test the prediction of the integrative encoding hypothesis using an explicit measure of false memory, in addition to measuring response bias and reaction time. We collected five datasets (experiments) using variations of the acquired equivalence paradigm, including a close replication of the procedures implemented by Shohamy and Wagner (2008). Data are reported jointly for clarity and brevity, noting any differences across datasets.

\section{Materials and methods}

Participants. A total of 197 participants were enrolled in the study. All participants were students enrolled in an undergraduate program at the University 
of Oregon, age 18-35, who had normal or corrected-to-normal vision and gave informed consent. Seven participants who did not reach $60 \%$ on trained associations (chance 50\%), which was more than 3 standard deviations below the rest of the group, were excluded from further analyses, for a final sample size of 190. The full sample consists of five datasets, collected while varying details of the procedures to test the robustness of the results. The number of participants in each Dataset and their demographic information are reported in Table 1.

Table 1. Demographic information for each Dataset.

\begin{tabular}{|l|l|l|l|}
\hline Dataset & N included (excluded) & Age range (mean) & Gender \\
\hline 1 & $39(1)$ & $18-29(19.0)$ & 23 females; 16 males \\
\hline 2 & $37(3)$ & $18-27(20.3)$ & 28 females; 9 males \\
\hline 3 & $40(0)$ & $18-32(20.2)$ & 29 females; 11 males \\
\hline 4 & $37(0)$ & $18-28(19.3)$ & 22 females; 15 males \\
\hline 5 & $37(3)$ & $18-29(21.1)$ & 27 females; 10 males \\
\hline
\end{tabular}

Procedure. The paradigm was a close behavioral replication of the acquired equivalence study by Shohamy and Wagner (2008). A formal source memory test was added as a final phase to explicitly test how well participants differentiate whether they directly learned or inferred a piece of information and whether source memory and generalization are related in this paradigm. Participants were not informed about the final source memory test until the completion of the previous phases, making the results of the training and generalization phases directly comparable to prior work. Thus, any differences between current results and prior work cannot be due to the addition of the source memory test. While all tests were identical across Datasets, stimuli and training procedures varied between Datasets, as described below.

Stimuli. The stimuli and procedures were modeled after Shohamy and Wagner (2008). The stimuli were 24 grayscale images of faces and 24 colored images of scenes, randomly assigned into 12 quadruplets, different for each participant. Each quadruplet included 2 faces (face pairmates Face1 and Face2), associated with 2 scenes (Scene1 and Scene2). There were four possible facescene associations within each quadruplet: Face1-Scene1, Face1-Scene2, Face2-Scene1, Face2-Scene2. Three of these face-scene associations were used for training (Face1-Scene1, Face1-Scene2, and Face2-Scene1), the last one (Face2-Scene2) was subsequently used as a test of generalization via acquired 
equivalence (Figure 1). Starting with Dataset 2, only Caucasian faces were used to minimize other-race effects in our predominantly Caucasian sample, and the face pairmates were limited to consist of one male and one female (Preston et al., 2004) to further minimize item effects.

Pre-exposure. Participants first passively viewed the 24 face stimuli, each presented three times in random order, to become familiar with them. Faces were presented one by one for $2 \mathrm{~s}$ with a $1 \mathrm{~s}$ fixation cross in between. While Shohamy and Wagner (2008) included a manipulation during pre-exposure, presenting only a subset of stimuli, they found no effect of this manipulation and report data collapsed across all stimuli. We thus pre-exposed all faces.

Training. During training, participants learned three face-scene associations (Face1-Scene1, Face1-Scene2, and Face2-Scene1) from each quadruplet, using feedback-based training. In each trial, participants were presented with a probe face on the top of the screen, and two choice scenes (a correct scene and a foil scene) below it. The foil was a scene from a different quadruplet. Each quadruplet was yoked to another so that both quadruplets had the same scene options (Scene 1, Scene 1', Scene 2, and Scene 2'), but differed in scene preference: scenes from one quadruplet always served as foil scenes for faces from the other quadruplet and vice versa. This ensured that participants had to base their response on memory for the correct face-scene associations.

The face and the two choice scenes stayed on the screen for $3 \mathrm{~s}$, a time during which the participant had to choose a scene preferred by the face probe. When the 3s had elapsed, participants received feedback ('Correct', 'Incorrect', or 'Too late') that stayed on the screen for $1 \mathrm{~s}$, followed by a $1 \mathrm{~s}$ fixation cross. Each association was presented 8 times, for a total of 288 trials (12 quadruplets $x$ 3 trained associations in each $x 8$ repetitions) in a randomized order. The correct scene was presented an equal number of times on the left side and on the right side of the screen. Participants received a self-paced break half-way through training.

Training procedure differences between Datasets. Datasets 1 and 2 were collected using identical procedures, matching those of Shohamy and Wagner (2008). The only difference between the Dataset 1 and Dataset 2 was that face stimuli in Dataset 1 were of mixed races, paired randomly. As noted above, Dataset 2 (and all following Datasets) limited face stimuli to Caucasian faces and pairings to one female and one male face, to minimize item effects. In Dataset 3, we employed a commonly used scaffolding presentation in which related 
associations are introduced gradually to facilitate learning (Collie et al., 2002; Doll et al., 2015; Honey \& Hall, 1991; Meeter et al., 2009). Translating this scaffolding method to our paradigm with faces and scenes, one scene preference for one face from a quadruplet is repeatedly trained (Face1-Scene1). Then, the same scene preference is introduced for the face pairmate (Face2-Scene1) to indicate preference equivalence across the two faces. Third, the second scene preference for the first face is introduced (Face1-Scene2). Finally, all training trial types are intermixed during the last block of training. For Dataset 4, training trials were again presented in random order but the number of training repetitions was increased from 8 to 12. For Dataset 5 , the training also consisted of 12 repetitions but was administered about 24 hours before the acquired equivalence and source memory test phases.

Acquired equivalence test. After training, participants underwent an acquired equivalence test without feedback. They were tested on the associations learned during training (Face1-Scene1, Face1-Scene2, and Face2-Scene1), as well as on the untrained face-scene association (Face2-Scene2) (Figure 1). Stimuli remained on the screen for $3 \mathrm{~s}$ and were separated by a $1 \mathrm{~s}$ fixation cross. Following Shohamy and Wagner (2008), each association was repeated 6 times to obtain a more robust estimate of preference, resulting in a total of 288 trials (12 quadruplets $\times 4$ associations in each $\times 6$ repetitions), randomly intermixed. As in training, correct scenes were presented on each side of the screen an equal number of times. If the participants generalized across pairmates, they should show a response bias on untrained trials. For example, if Face1 prefers Scene2 over Scene2', participants may also systematically pick Scene2 over Scene2' for Face2. If participants did not generalize across the faces, they would not have a systematic response bias on untrained trials. The response bias, response times for trained and untrained face-scene associations, and the difference between those response times were the main measures of interest. For simplicity, and to be consistent with Shohamy \& Wagner (2008), we will usually refer to the response bias scores as "generalization", even though the findings indicate that participants did not actually generalize often.

Source memory test. A source memory test was included to explicitly test the hypothesis that generalization may lead to source memory confusion and false memory. On each trial, participants were presented with one face and two scenes, arranged vertically above each other to remove the left-right organization of scenes. The task was to indicate if and when the three pictures were presented together previously, irrespective of their spatial arrangement. There were four 
possible answer options. "Training only" would indicate having seen the three images together, but only during the training. This answer was never correct and was included so that the choices did not convey information about the structure of the task. "Test only" would indicate that they had seen the three images together, but only during the acquired equivalence test. This answer was correct for the untrained associations (Face2-Scene2-Scene2'). "Both" would indicate they had seen the three images together during both training and the acquired equivalence test. This answer was correct for all the trained trials (e.g., Face1Scene1-Scene1'). Observing increased incidence of responding "both [training and test]" to untrained pairs, especially in those participants with strong generalization scores, would substantiate the anecdotal evidence from Shohamy and Wagner (2008) regarding the postulated trade-off between generalization and episodic memory details. Thus, false memory (\% response "both" for untrained trials) was the main measure of interest from the source memory test.

\begin{tabular}{|l|c|l|l|}
\hline $\begin{array}{l}\text { Trial type } \\
\text { condition }\end{array}$ & N trials & Description & $\begin{array}{l}\text { Correct } \\
\text { answer }\end{array}$ \\
\hline Trained & 36 & $\begin{array}{l}\text { Triplets presented during training and } \\
\text { acquired equivalence test }\end{array}$ & Both \\
\hline Untrained & 12 & $\begin{array}{l}\text { Triplets presented only during the } \\
\text { acquired equivalence test }\end{array}$ & Test only \\
\hline $\begin{array}{l}\text { Recombined- } \\
\text { scenes }\end{array}$ & 12 & $\begin{array}{l}\text { Each scene had been presented with the } \\
\text { face, but the two scenes had not been } \\
\text { presented together }\end{array}$ & Never \\
\hline $\begin{array}{l}\text { Recombined- } \\
\text { face }\end{array}$ & 12 & $\begin{array}{l}\text { Two scenes which were presented } \\
\text { together during training but with a } \\
\text { different face }\end{array}$ & Never \\
\hline Recombined-all & 12 & $\begin{array}{l}\text { A face and two scenes which were never } \\
\text { presented together }\end{array}$ & Never \\
\hline
\end{tabular}

Table 2. Types of trials included in the source memory test

The last response option was "Never," indicating that the three images had not been presented together on a single screen and instead the images were recombined in some way. We have constructed three types of recombined triplets for which "Never" was the correct answer: Recombined-face 
combinations included triplets of two scenes presented together previously, but with a different face (e.g., Face1-Scene3-Scene3'). Recombined-scenes combinations included triplets of a face and two scenes that had been presented with the face but not with each other (e.g. Face1-Scene1-Scene2).

Recombined-all triplets included a face and two scenes in which no two were ever presented together in previous phases (e.g., Face1-Scene3-Scene5). The source memory test included twelve trials of each recombined-face, recombinedscenes and recombined-all triplets. Inclusion of these recombined source memory trials allowed us to control for any response bias and see whether false memory responses are unique to the untrained trials. A summary of all trial types in the source memory test is presented in Table 2.

Generalization-source memory relationship. To test the relationship between generalization and source memory, we employed two analyses. First, we performed an explicit test of the anecdotal evidence from Shohamy and Wagner (2008) regarding a lack of source memory in participants who generalized. Presumably, participants who had formed an integrated representation linking faces and scenes within a quadruplet together may have been unaware that some of those associations had not been trained. During the source memory test for untrained pairs, they would be less likely to respond "test only" and more likely to respond "both" (false memory). Participants who formed separate representations of the trained association may have lower generalization scores and respond slower on the untrained pairs, but should be aware during the acquired equivalence test that the untrained pairs had not been studied previously, showing low false memory. Thus, false memory was compared between participants who displayed a consistent response bias in the direction of acquired equivalence ("good" generalization), those who did not show a consistent response bias ("poor" generalization), and those who showed a consistent response bias but in the opposite direction. We also used correlation between the response bias score (treated as continuous variable) and false memory scores to verify that results were not driven by dichotomizing generalization scores.

As a second analysis, we tested whether generalization across faces predicted false memory on a trial-by-trial basis, conceptually replicating analysis by Carpenter and Schacter $(2017,2018 \mathrm{a})$. First, the 12 untrained relationships for each participant were sorted based on whether the participant (a) showed a consistent response bias for a given untrained relationship in at least 5 out of 6 test trials, (b) had consistent response bias away from acquired equivalence (consistently picked the scene not associated with its pairmate on at least 5 out of 6 test trials), or (c) did not show consistent bias for that untrained trial (each 
scene picked 2-4 times). We then compared false memory for the three trial types (positive bias trials, negative bias trials, no bias trials) using a repeated measures ANOVA. As there were only 12 pairmates per participant, split into three conditions for the trial-by-trial analyses, the analysis is underpowered withinsubject. Furthermore, participants who did not have at least one trial of each type were excluded from this analysis. To increase power and ensure we did not miss any relationship that may be present, we performed two additional control analyses. In one, we excluded the lowest-count negative bias trials from the analysis and only compared generalized vs. not-generalized trials, retaining more participants in the analysis. In the second control analysis, we used a hierarchical linear regression model that included all subjects, treated as a random factor. However, the given the low trial counts per trial type, all trial-by-trial analysis should be considered exploratory.

\section{Results}

Memory for trained preferences and response bias on untrained preferences (generalization)

Memory for trained associations and generalization scores were relatively consistent across datasets (one-way ANOVA, both $\mathrm{F}<1.24, \mathrm{p}>$.29; Figure 2A, B) and were aggregated for subsequent analyses. Participants remembered about $87 \%$ (SD $=9.4 \%$ ) of trained preferences. On untrained trials, participants showed a small response bias consistent with acquired equivalence indicative of generalization ( $M=55 \%, S D=14.9 \%$ ), which was reliably different from a theoretical no-bias score of $50 \%(t(189)=4.72, p<0.001, d=0.34)$. The mean response bias of $55 \%$ was substantially lower than $81 \%$ reported by Shohamy and Wagner (2008), although within the $50-70 \%$ range reported in other acquired equivalence studies (Doll et al., 2015; Duncan et al., 2012; Foerde \& Steinglass, 2017). Thus, across five independent datasets, we found above-chance tendency to generalize across pairmates, but the degree of generalization was relatively modest. 
A

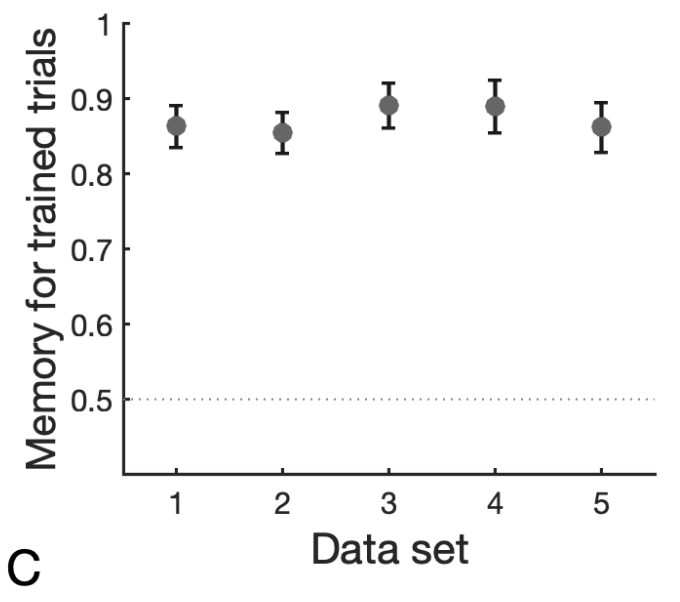

B

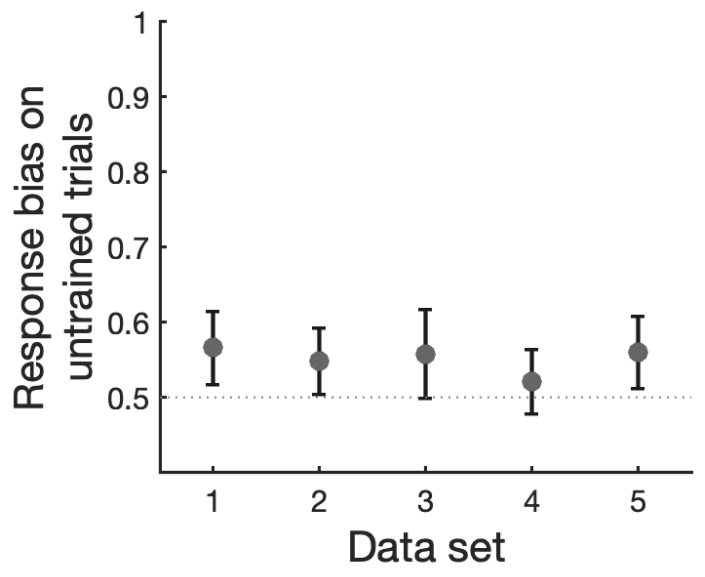

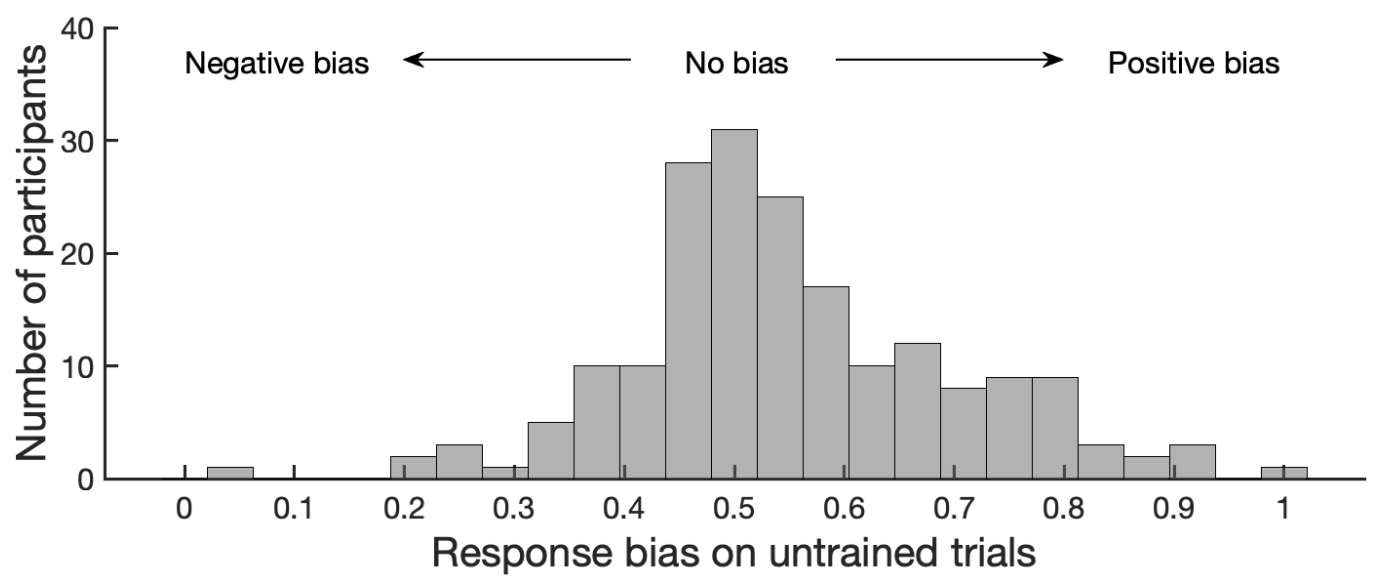

Figure 2. Memory for trained preferences and response bias on untrained preferences (generalization). A. The average proportion of trained preferences that participants correctly remembered at test, separately for each dataset. Dataset 1 was from a direct behavioral replication of Shohamy \& Wagner, 2008. Dataset 2 followed identical procedures but with different face stimuli. Dataset 3 included scaffolded training to facilitate learning. Dataset 4 included 12 rather than 8 repetitions of each pair. Dataset 5 included 12 repetitions and a delayed test. See Methods for more details. $\boldsymbol{B}$. The average response bias on untrained trials, separately for each dataset. In both A \& B, error bars denote the $95 \%$ confidence interval for the mean. Dotted line denotes chance/no response bias. $\boldsymbol{C}$. The distribution of response bias scores on untrained trials across all Datasets. Response bias scores near .5 indicate no consistent response bias. High scores indicate a positive response bias in the direction of acquired equivalence. Low scores indicate a negative response bias where a participant tended to select a scene for a face that was not preferred by their pairmate.

As the $87 \%$ trained pairs accuracy was also lower than $93 \%$ trained accuracy observed in Shohamy and Wagner (2008), we wanted to test whether generalization may be higher when considering only subjects or trials where the trained pairs are remembered to a high degree. Thus, we performed two control analyses. First, we only included untrained trials for which a given participant 
reliably remembered all trained associations (at least 5 correct responses out of 6 repetitions of each of Face1-Scene1, Face1-Scene2 and Face2-Scene1 pairs), obtaining a mean generalization score of $59 \%$ (SD = 18.2\%). Second, we excluded all participants who scored below $80 \%$ on trained pairs to obtain a subsample $(N=144)$ that had a similar performance on trained pairs $(M=92 \%, S D=$ $4.9 \%$ ) as that found in Shohamy and Wagner (2008). This sub-sample showed an average generalization score of $57 \%(S D=15.8 \%)$. Thus, even when we focused only on well-remembered trials or on participants with good memory for trained associations, response bias remained under $60 \%$.

\section{Distribution of generalization scores}

Given the low mean generalization scores, we wanted to determine whether individual participants typically do not show any response bias and respond around 50/50 for each scene, or whether they do show a bias but in inconsistent directions. We thus examined the distribution of individual scores (Figure 2C). We found that most participants did not have a response bias on untrained trials and were equally likely to choose a scene associated with the face's pairmate as the other scene option (response bias scores near 0.5 , reflecting a lack of generalization). However, the number of participants that showed a consistent response bias in one or the other direction was greater than chance. Based on the binomial test (72 untrained trials at test, probability of choosing each scene by random $p=.5$ ), response bias above .6 should be observed with less than $5 \%$ probability by chance. Yet, 57 participants $(30 \%)$ had a reliable response bias consistent with generalization through acquired equivalence. Response bias under .4 should be also observed with less than $5 \%$ probability by chance. Yet, 22 participants (12\%) had a reliable response bias in the direction opposite to acquired equivalence, consistently picking the scene not associated with a pairmate. The observed counts in each bin (22 negative response bias, 111 near chance/no bias, 57 positive response bias) were reliably different from counts that would be expected if everyone responded randomly (expected: 9.5 seemingly negative bias, 171 no bias, 9.5 seemingly positive bias; $\chi^{2}(2)=275, p<.001$ ). Thus, it appears that a portion of participants showed a consistent response bias, although some of those participants consistently showed a response bias away from acquired equivalence. This may reflect a repulsion or pattern differentiation of overlapping memories as a protection from interference (Chanales et al., 2021; Hulbert \& Norman, 2015). The proportion of participants showing a positive response bias (i.e., generalization across pairmates) was reliably greater than those showing a negative response bias $\left(\chi^{2}(1)=15.5, p<0.001\right)$. The three groups of participants (no bias, positive bias, negative bias) also differed in how 
well they remembered the trained associations (one-way ANOVA $F(2,187)=6.21$, $p=0.002)$. This was driven by lower memory for trained preferences in those who showed no response bias $(M=86 \%, S D=9.9 \%)$ or a negative response bias ( $M$ $=85 \%, \mathrm{SD}=9.6 \%)$ than those who showed a positive response bias $(\mathrm{M}=91 \%$, $\mathrm{SD}=7.1 \%$, both $\mathrm{t}>2.7, \mathrm{p}<0.008$ ).

\section{Response times and their relationship to generalization}

It would be expected that participants respond faster on trained trials than on untrained trials during the test. However, a striking pattern of response times was found in Shohamy and Wagner (2008), with minimal response time cost on untrained trials observed for those who showed high degree of generalization. This finding provided a strong support for the conclusion that links between related events had been formed at encoding and the untrained relationships were already represented in memory just as directly learned associations.
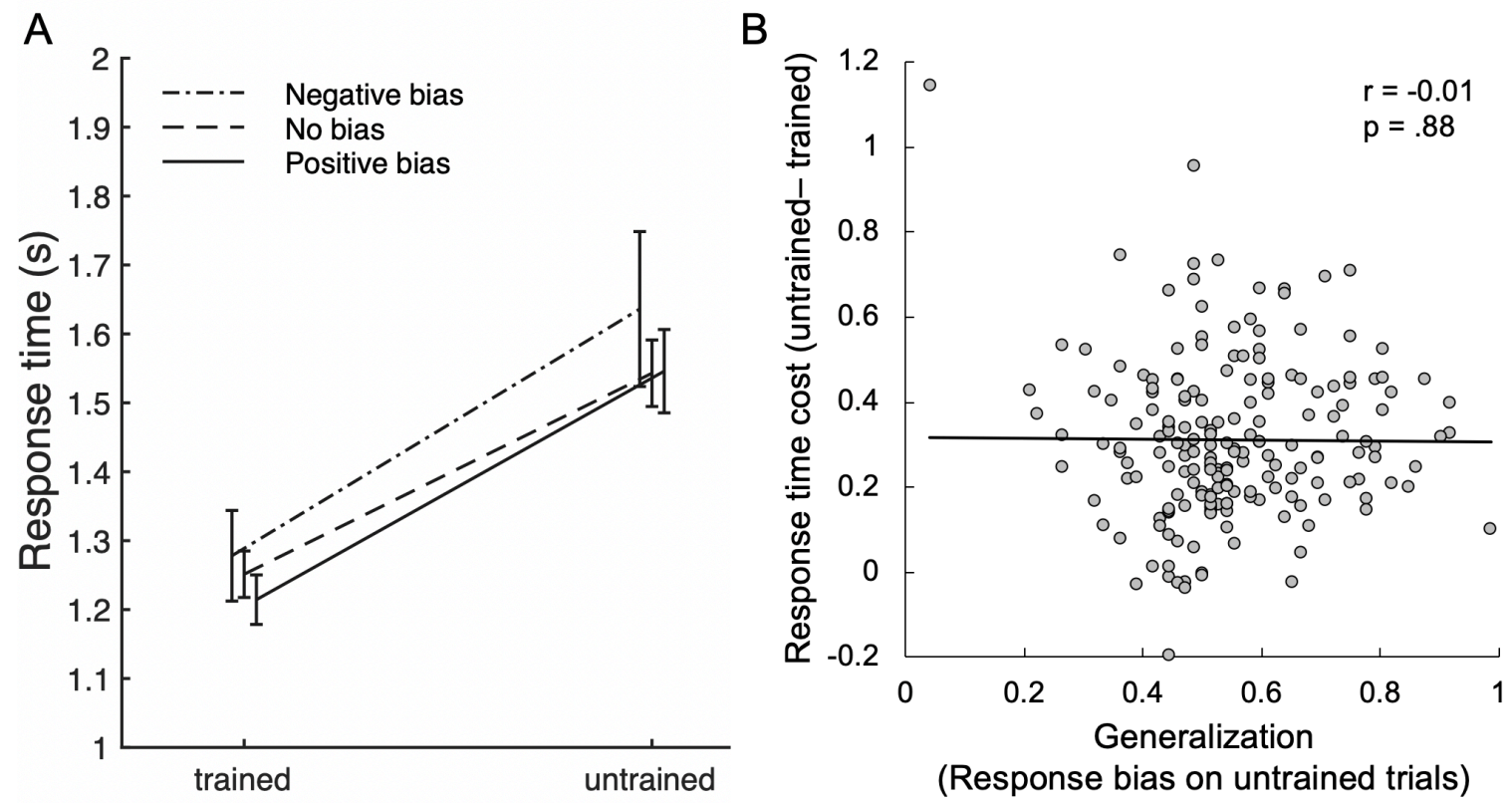

Figure 3. Relationship between reaction time and generalization. A. Treating participants with different response bias as distinct groups. Average of median response times on trained and untrained trials, separately for participants that showed negative response bias, no bias, and positive response bias (generalization). Error bars denote $95 \%$ confidence interval of the mean. $\boldsymbol{B}$. Treating response bias as a continuous variable. Each dot represents a participant.

This pattern was not replicated in the current data (Figure 3). For each participant, we computed the median response time for correctly remembered trained trials and the median response time for "correct generalization", or untrained trials on which the participant made a response consistent with acquired equivalence. In the first analysis, depicted in Figure 3A, we treated participants' response bias as a categorical variable. Specifically, we compared 
the response time cost among participants who showed a positive response bias ("good generalization" group in Shohamy and Wagner, 2008), no bias ("poor generalization" group in Shohamy and Wagner, 2008), and negative response bias (not considered in Shohamy and Wagner, 2008). A mixed-design ANOVA with trial type (trained, untrained) and response bias group (negative bias, no bias, positive bias) showed a main effect of trial type, with longer response times on untrained $(M=1.56 \mathrm{~s}, S D=0.25 \mathrm{~s}$ ) than trained trials (trained $M=1.24 \mathrm{~s}, \mathrm{SD}=$ 0.16 s: $F(1,187)=373, p<0.001)$. There was no effect of response bias group $(F(2,187)=1.36, p=0.26)$ and no group * trial type interaction $(F(2,187)=1.59, p$ $=0.21$ ), with a response time cost around $300 \mathrm{~ms}$ for all groups. No reduction of response time cost was seen even when considering only participants with the highest generalization score above $.80(N=12$, response time cost $=337 \mathrm{~ms}, \mathrm{SD}$ $=127 \mathrm{~ms}$ ).

Treating response bias as a categorical variable allowed us to compare "good" and "poor" generalizers, replicating the analysis from Shohamy \& Wagner (2008), while also explicitly separating participants who showed a negative response bias for whom we did not have a clear response time prediction. To verify that the results were not affected by treating the continuous response bias scale as categorical, we conducted a control analysis using an across-subject correlation of the continuous measures of response time cost (untrained minus trained) with continuous generalization scores. Integration at encoding should lead to higher generalization scores accompanied by smaller reaction time cost on untrained trials. In line with this prediction, a strong negative correlation of $r=-$ 0.69 was observed by Shohamy and Wagner (2008). However, no such correlation was found in the current data (Pearson $r=-0.01, p=.89$; Spearman $r$ $=0.09, p=.23$; Figure 3B). Excluding participants with negative response bias who may have had a different approach to the task -also did not produce the expected negative correlation and instead produced a small positive correlation (Pearson $r=0.13, p=0.092$; Spearman $r=0.17, p=0.03$ ). When split across datasets, the only reliable and robust correlation was observed in Dataset 5 (Pearson $r=0.34, p=.04$; Spearman $r=0.42, p=0.008$ ) and it was positive, in the opposite direction than predicted. Participants who showed the greatest degree of generalization did so at a higher reaction time cost, rather than a lower reaction time cost. In Datasets 1, 2 and 4, there were no reliable correlations (all Pearson and Spearman $r$ s between [+0.02 and +0.16], all ps > .3). In Dataset 3, we observed the only correlation in the predicted negative direction (Pearson $r=-$ $0.37, p=.02$; Spearman $r=-0.21, p=.19$ ). However, the correlation was driven by an outlier with an extreme negative bias $(z=-2.8)$ and an extreme reaction time cost $(z=3.8)$; see the data point in the upper left corner of Figure 3B. 
Without this participant, the correlation dropped to $r=-0.13(p=.42)$. The lack of compelling evidence for a negative correlation is unlikely due to low power. If there was a moderate negative relationship $(r=-0.5)$, we would have over $90 \%$ power to observe it within each dataset. Even a modest negative relationship ( $r=$ -0.2 ) would be detectable with over $80 \%$ power in the full sample. To summarize, contrary to our prediction and contrary to the integrative encoding hypothesis, we found no evidence that higher generalization scores would be associated with lower response time costs for untrained trials.

\section{Linking memories during test}

So far, the results suggest that participants did not make links across related experiences often. We wondered whether the links that were formed may have resulted from task demands at retrieval rather than at encoding (Banino et al., 2016;

Carpenter \& Schacter, 2017, 2018a). For example, the REMERGE model postulates that links between indirectly related elements can be made through recurrent activation of directly related elements, without the need to postulate that memories are integrated into a combined

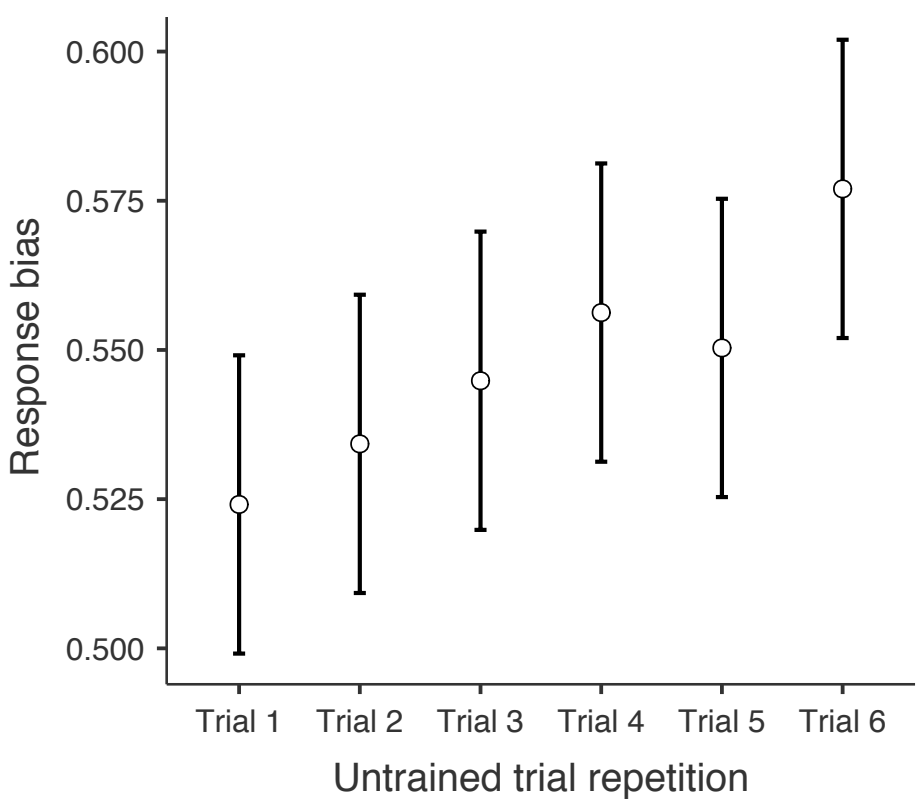
representation (Banino et al., 2016;

Figure 4. Mean response bias on untrained trials as a function of repetition during test. Error bars denote 95\% confidence interval of the mean.

Kumaran, 2012; Kumaran \& McClelland, 2012a). One consequence of such a mechanism would be strengthening of the links between indirectly related elements over repeated testing, as they would be repeatedly co-activated through the recurrent connections, and then re-encoded back into the hippocampus (Koster et al., 2018). We thus looked at the degree of response bias separately in each of the 6 test repetitions of untrained trials. We found that the average response bias increased across test repetitions (repeated measures ANOVA F $(4.1,774)=5.81, p$ $<0.001$, Greenhouse-Geisser corrected; linear trend $F(1,189)=15.0, p<0.001)$, from $52.6 \%$ on the first untrained trial to $58.2 \%$ on the last untrained trial (Figure 4). Similar results were observed across all 5 datasets (trial * version interaction $F$ $<1, p>0.8)$. Thus, participants continued to make links across related memories during the test, consistent with what would be predicted by REMERGE. 
Importantly, no changes in performance were seen across repeated testing of the trained associations (range $86.6 \%-87.6 \%$, repeated measures ANOVA and linear trend both $\mathrm{F}<1$ ) as no additional learning can happen on those trials without feedback, nor those associations can be inferred from other associations. Thus, the increase in generalization scores across repeated testing is unlikely due to non-specific factors (such as motivation), changing across time.

\section{Source memory}

When participants spontaneously link related events into an integrated memory representation, they may not realize they are making a novel judgment on untrained trials. This was previously reported anecdotally (Shohamy \& Wagner, 2008), but not yet tested empirically in this paradigm. A novel aspect of the current study was the addition of an explicit source memory test, administered after the generalization test in order not to alert participants to the fact that some of the test trials were untrained. It also allowed us to compare our generalization results with those reported previously (e.g., Doll et al., 2015; Duncan et al., 2012; Shohamy and Wagner, 2008). On each source memory trial, participants were shown a face and two scenes and asked whether and when they saw those stimuli together. This allowed us to explicitly measure the degree of false memory of having seen the untrained trials in the training phase. Participants had four response options regarding when they have seen each face-scene-scene combination: study only (never correct), test only (correct for untrained), both study and test (correct for trained) or never (correct for recombined trials). The mean proportion of each of four possible response options given to each trial type is depicted in Figure 5. On average, participants tended to remember whether and when they had seen a face and two scenes together (overall accuracy $\mathrm{M}=74 \%, \mathrm{SD}=13.7 \%)$. On untrained trials, participants most often correctly recognized them as seen at test only ( $M=63 \%, S D=27.5 \%)$. The main measure of interest from the source memory test was the degree of false memory, i.e. the proportion of "both study and test" response for untrained trials. False memory of having seen untrained trials during both study and test occurred on the average of $27 \%$ of trials (SD $=24 \%$ ), but it was not a dominant response for any one participant. Rather, a single participant chose equally frequently "test only" and "both study and test" for untrained trials. All remaining 189 participants choose the correct "test only" option more often than the integration-predicted (false memory) option. Thus, all participants were mostly aware that they had not studied the untrained trials. When split across datasets, the average false memory scores ranged between $21 \%$ to $33 \%$ (one-way ANOVA $F(4,185)=1.25, p$ $=0.29$ ), 


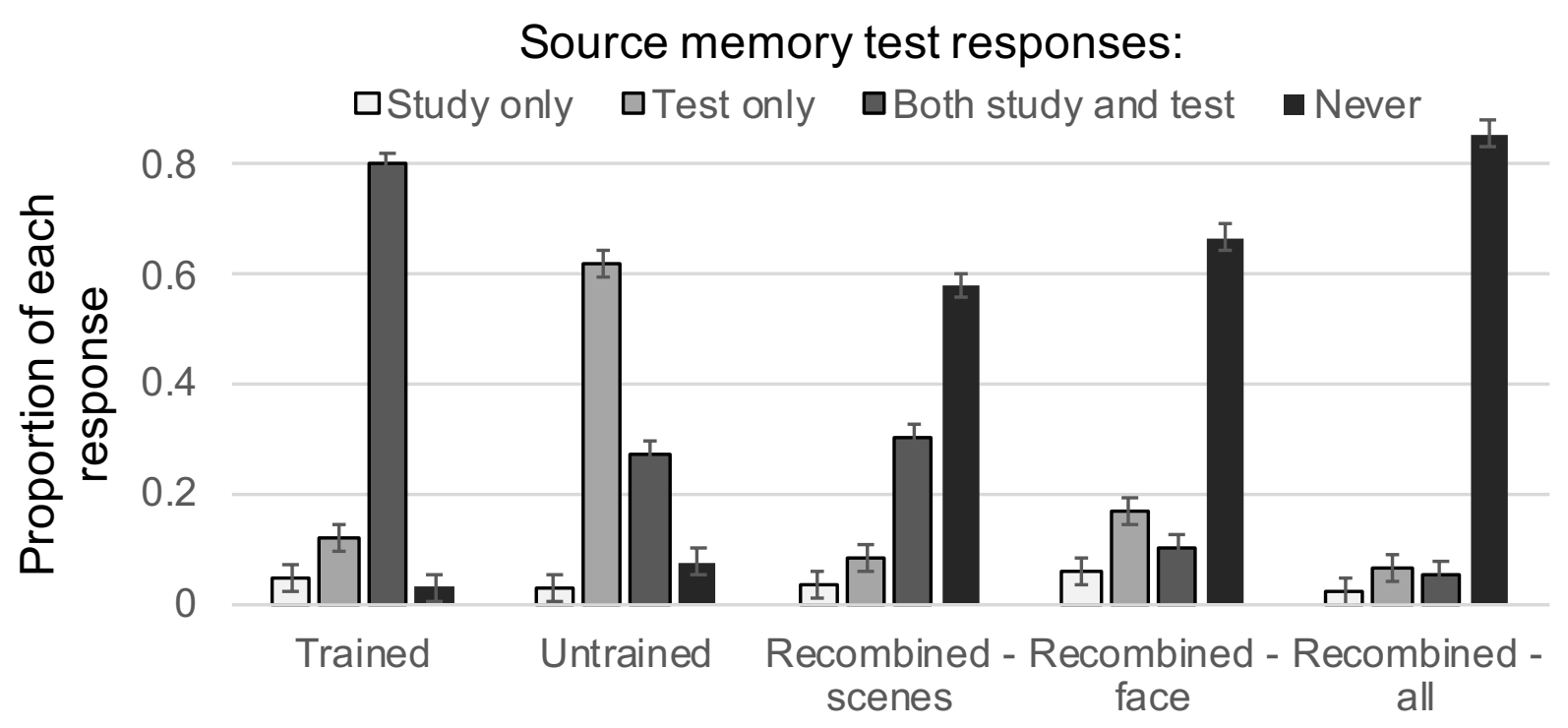

Figure 5. Mean proportion of each response for each trial type in the source memory test. Error bars denote $95 \%$ confidence interval of the mean.

\section{False memory and generalization}

Next, we tested the hypothesis that false memory for inferred relationships may be a flip side of memory integration (Carpenter \& Schacter, 2017; Shohamy \& Wagner, 2008; Varga et al., 2019). This hypothesis predicts a positive relationship between generalization and false memory for untrained trials. First, we used across-subjects correlation to test whether participants who showed greater generalization tendencies also showed higher false memory rates. We instead found non-significant, numerically negative correlation (Pearson $r=-$ $0.106, p=.146$; Spearman $r=-0.123, p=0.08$; Figure $6 A$ ), meaning that "good" generalizers were more likely to show decreased rather than increased false memory. Similar results were found in all datasets, with correlations ranging between $r=-0.28$ to +0.03 . Furthermore, no across-subject relationship between generalization and false memory emerged when treating participants' generalization tendency as a categorical variable, comparing false memory rates in participants who showed a negative response bias, no bias or a positive response bias $(F(2,187)=0.75, p=.48)$. Thus, we found no evidence that generalization and false memory would go hand-in-hand. 

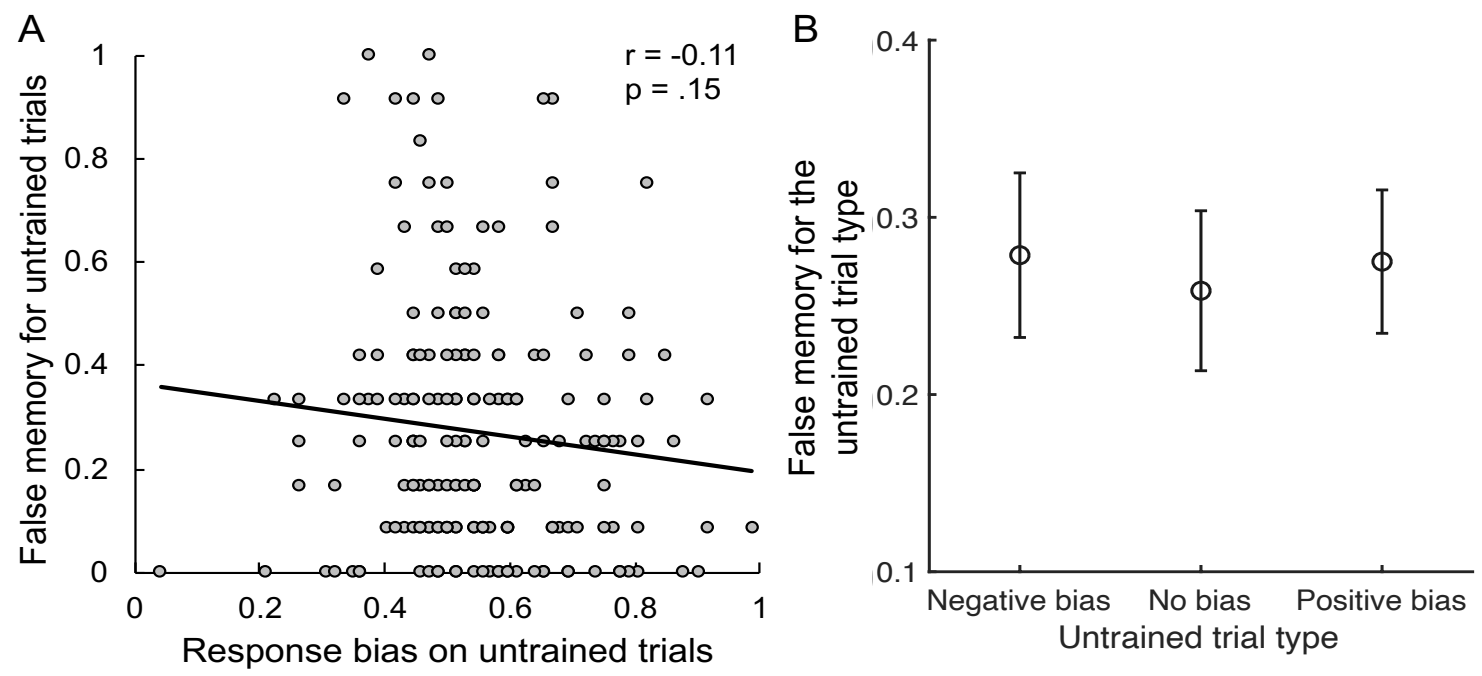

Figure 6. Relationship between generalization and false memory. A. Across-subject analysis. Correlation between generalization scores and false memory scores. B. Within-subject analysis. Mean false memory rates on untrained trials for which participants consistently chose the option NOT associated with the pairmate (negative bias), chose each response option about equally (no bias), and consistently chose the option associated with the pairmate (positive bias/generalization). Error bars denote 95\% confidence interval of the mean.

As a second approach, we focused on within-subject effects and conceptually replicated trial-by-trial analyses by Carpenter and Schacter $(2017$, 2018a). This analysis considers that links between related events may happen for some events but not others. False memory would only emerge for those untrained trials on which a given participant linked the corresponding trained trials and exhibited generalization. Thus, for each participant, we sorted their untrained associations (F2-S2) to those for which the participant (1) showed a consistent generalization response bias and selected the scene that was the pairmate's preference on at least 5 out 6 repetitions ('positive bias'), (2) showed a consistent negative response bias and selected the scene that was not the pairmate's preference on at least 5 out of 6 repetitions ('negative bias'), and (3) untrained associations with no clear response bias ('no bias'). Given that there were only 12 untrained pairs per participant that were binned into these three trial types (consistent positive bias, consistent negative bias, no bias), this analysis is underpowered within-subject and should be interpreted with caution.

Nevertheless, we wanted to explore all analytical approaches that could uncover any potential relationship between generalization and false memory. Repeatedmeasures ANOVA in a subset of participants $(N=163)$ that had at least one trial of each type did not show reliable differences in false alarm rates based on the trial's response bias status (ANOVA $F(2,324)=0.55, p=.57$; Figure $6 B$ ). Similar 
results were observed when we only focused on positive response bias vs. no response bias trials $(N=177, t(196)=1.09, p=0.28)$. Finally, to better account for the varied number of trials going into each response bias bin in each subject, we employed a mixed-effect model, modeling trial-by-trial probability of a false memory response as a function of response bias for the given pairmate, with subjects and dataset as random effects. We did not observe an overall effect of trial-by-trial response bias $(F=0.81, p=0.44)$ nor any pair-wise differences between response bias conditions (each pair-wise contrast across the negative bias, no bias, positive bias trial type conditions, all $F<1.45, p>$.2). Thus, linking related events, as evidenced by a positive response bias at the level of participants (Figure 6A) or trials (Figure 6B), did not appear to be associated with false memory for the inferred associations in our data.

\section{False memory and reaction time cost for untrained trials}

While we hypothesized that generalization and false memory may be flip sides of the same coin, both resulting from memory integration, we did not such a relationship when looking at generalization success. However, both integration at encoding and retrieval-based inference can lead to generalization response at test, but only integrationbased generalization would be thought to relate to false memory. As our results indicate that retrieval-based processes likely contributed to generalization decisions in the current study, we were wondering whether reaction time may be a better marker of integration than generalization score alone. Specifically, integration would be expected to lead to faster generalization responses than retrievalbased inference (Schlichting et al., 2014; Shohamy \& Wagner, 2008).

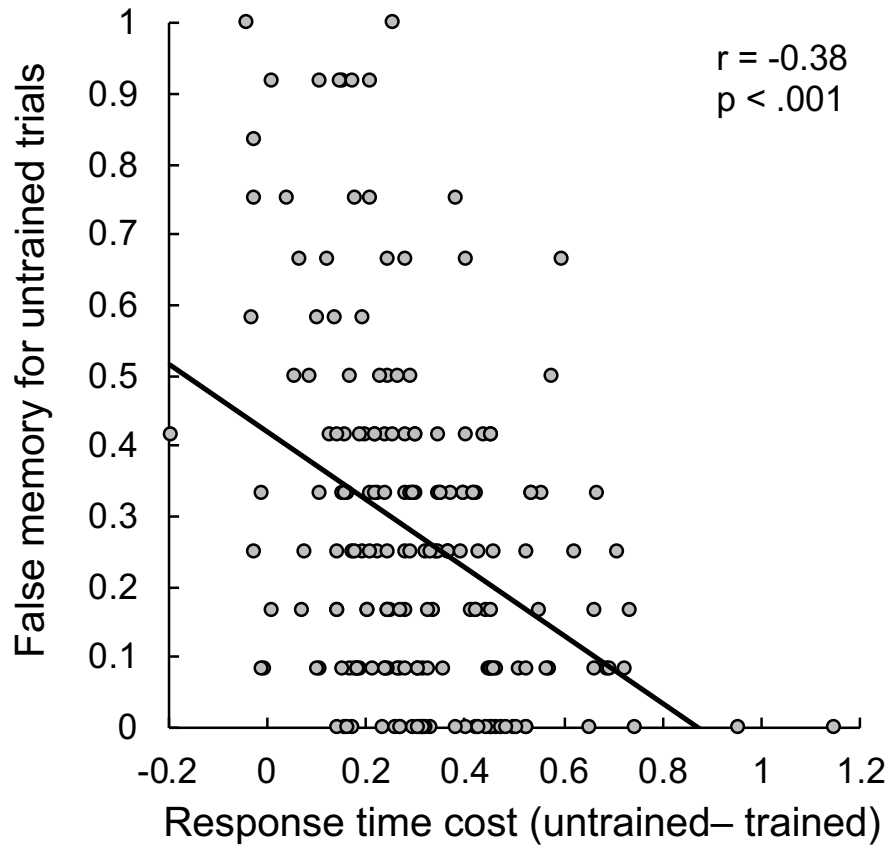

Figure 7. Correlation between response time cost for untrained trials during the generalization test and false memory for having seen untrained trials during both study and test, as reported during the subsequent source memory test. 
To test this idea, we corelated response time cost at generalization test (untrained minus trained) with the degree of false memory for untrained trials at subsequent source memory test. Consistent with the integration prediction, we found a reliable negative correlation, such that participants who showed minimal RT cost for untrained trials were more likely to falsely remember seeing the untrained trials at both study and test (Pearson $r=-0.38, p<0.001$; Spearman $r=$ $-0.36, p<0.001$; Figure 7). In other words, as suggested by Shohamy and Wagner (2008), it appears that integrating related memories afforded some participants a speed advantage on generalization trials but at the expense of memory specificity, resulting in an increased tendency to confuse inferred relationships for observed ones.

\section{Discussion}

Memory allows us to remember details of specific events, such as how we encountered a piece of information. It also allows us to transcend individual events and link pieces of information acquired at different times to generalize and infer new information. How generalization is accomplished has been a matter of debate. Single system accounts propose that generalizations can be computed on-the-fly from specific memories at retrieval, without a need for generalized memory representations per se (Kumaran \& McClelland, 2012a; Nosofsky, 1986). Other theories assume that memory specificity and generalization are based on distinct types of representations (Minda \& Smith, 2011; Posner \& Keele, 1968; Schlichting \& Preston, 2017). Generalized representations that combine elements across events can be formed via reactivation and integration of related events during encoding (Ashby et al., 2020; Shohamy \& Wagner, 2008; Zeithamova, Dominick, et al., 2012), although it is also possible to encode related memories as separate and integrate them subsequently offline at rest (Barron et al., 2020) or in response to task demands (Carpenter \& Schacter, 2017, 2018a).

Here, we used an acquired equivalence paradigm with an added explicit source memory probe to test the hypothesis that generalization stems from a spontaneous integration of related memories. Unexpectedly, the results were aligned more with retrieval-based accounts of generalization than with integrative encoding predictions. Across five datasets collected using variations of the acquired equivalence paradigm, we found above-chance response bias on untrained trials that indicated participants generalized information across related experiences. However, generalization tendency was relatively small in magnitude (55\% compared to a chance of $50 \%$ ), with a majority of participants showing little generalization. Furthermore, the average generalization score was $52 \%$ the first 
time it was tested and increased linearly across repeated testing, indicating that participants continued making connections among related memories in response to task demands.

One model of generalization that could account for this pattern is REMERGE (Banino et al., 2016; Koster et al., 2018; Kumaran, 2012), which assumes that each event is initially stored as a separate representation but new inferences may emerge through recurrent connections when retrieval of one association triggers retrieval of a related association. One piece of evidence against the REMERGE model was a prior finding from acquired equivalence that generalization trials can be equally fast as directly learned trials, especially in those who show a high degree of generalization (Shohamy \& Wagner, 2008). This would indicate that links between related memories had been already made during encoding and no additional processing is needed at test. However, we did not replicate this striking reaction time pattern here. Instead, we found a reaction time cost for untrained trials that was present even in participants who showed the highest degree of generalization.

The increase in generalization throughout test repetitions also aligns with the REMERGE proposal that the retrieved output can be re-encoded back to the hippocampus (Koster et al., 2018). This way, initially separate elements can become linked in the hippocampus, once they were co-activated in response to task-demand. Similarly, single-cell recordings in primates found that regions showing no evidence of generalization during the first generalization trial may start representing the inferred relationships on subsequent test trials, as they encoded the output of the on-demand computation linking related memories at the first test trial (Pan et al., 2014). Thus, the increase of generalization scores throughout repeated testing observed here highlights the potential pitfall of averaging across test repetitions, when only the first trial may represent generalization per se (Pan et al., 2014; Taylor et al., 2021).

Another prediction tested here was the idea that integrating information across events may lead to false memory for the resulting inferences. Interestingly, a novel source memory test showed that participants were mostly aware that they were making novel judgments on untrained trials and did not mistake inferred relationships for observed ones often. Yet, false memory also occurred on about a quarter of untrained trials. We were curious whether false memory was related to generalization success, given the proposals that false memory and generalization are a flip sides of the same coin (Marsh et al., 2016; Shohamy \& Wagner, 2008; Varga et al., 2019). However, we did not find any such evidence. Participants who showed reliable generalization-consistent response bias on untrained trials-did not show greater false memory. On a trial-by-trial basis, false 
memory was also comparable for generalized and not-generalized untrained associations. Notably, because the false memory test was administered after the generalization test, we would be able to detect generalization-related false memory resulting from both integration during encoding (Shohamy \& Wagner, 2008) and on-demand integration at test (Carpenter \& Schacter, 2017, 2018a). Thus, the results indicate that generalization success does not always result in false memory. Rather, the source memory test results were again more aligned with retrieval-based accounts of generalization: as participants noticed that they were facing a novel probe at test, they perhaps engaged in an effortful, chained memory retrieval in an attempt to guess what the right answer may be, given their existing memories.

Notably, false memory and generalization are thought to be flip sides of the same coin because they both result from memory integration. However, generalization responses may be based on integrated memories or retrieval of separated memories of individual events (Schlichting et al., 2015; Zeithamova, Schlichting, et al., 2012; Zeithamova \& Bowman, 2020; Zeithamova \& Preston, 2010). With the contributing role of retrieval-based generalization, where generalization and memory specificity may go hand in hand rather than trade-off (Banino et al., 2016; Kumaran \& McClelland, 2012a; Nosofsky, 1986), generalization scores per se may not be a good proxy for integration in our data. We thus tested whether reaction times may provide a more sensitive index for integration. Specifically, generalization decisions based on integrated memories would be expected to be faster than those based on separate memories (Schlichting et al., 2014; Shohamy \& Wagner, 2008; Zeithamova \& Preston, 2017). Indeed, the response time cost predicted subsequent false memory, such that participants who showed smaller response time cost for untrained trials were more likely to report false memories for them. Presumably, at least some participants integrated related memories into a combined memory representation, which resulted in facilitated generalization accompanied by false memory for untrained trials, as would be predicted by the integrative encoding hypothesis (Shohamy \& Wagner, 2008).

Another novel observation from the current study was the tendency of some participants to bias their responses in the direction opposite from acquired equivalence. While participants with generalization scores below chance have been observed before (see e.g. Figure 2 in Doll et al., 2015), here our combined sample size provided a unique opportunity to verify that this effect is statistically reliable. One explanation for this finding may be that some participants represented related experiences in a manner that suppressed rather than encouraged the selection of pairmate-associated information. For example, 
overlap and similarity between memories often leads to interference (Keppel \& Underwood, 1962; Underwood, 1969), which can lead to repulsion or pattern differentiation of those memories (Chanales et al., 2021; Hulbert \& Norman, 2015). Perhaps greater vulnerability to interference may be associated with the tendency to exceedingly differentiate related experiences, which also aligns with the finding that reliable negative response bias was associated with somewhat worse memory for the premise pairs than reliable positive response bias. While these ideas are speculative at this time, future studies may provide additional insights into knowledge representation by considering the possible mechanisms behind such atypical response patterns. Nevertheless, the current findings highlight the varied behavioral consequences of event overlap on memory and suggest that representational strategies may vary across people.

How we connect bits and pieces of related information to form complex knowledge, infer new information, and generalize to new situations has been a question of much debate. The present study tested the degree to which people demonstrate decision biases through acquired equivalence and how well they retain specific information in this paradigm, testing predictions derived from current theories of generalization. The results demonstrate a role for both integrative encoding and retrieval-based inference in generalization, aligning with recent notions that no single answer is sufficient and multiple routes to generalization exist even in a single paradigm (Taylor et al., 2021; Zeithamova \& Bowman, 2020). These results align with recent evidence for both separated and integrated representations supporting generalization judgments under different circumstances, reported in both episodic inference and concept generalization (Bowman et al., 2020; Mack et al., 2013; Schlichting et al., 2015; Zeithamova \& Preston, 2017). Our results also highlight that related information is not always related in memory. Indeed, temporal proximity, strength of pre-existing memories, explicit instruction or individual differences have been all shown to affect whether related events are separated or integrated in memory (Cai et al., 2016; Carpenter \& Schacter, 2018b; Rashid et al., 2016; Richter et al., 2016; Schlichting et al., 2015; Tse et al., 2007; Zeithamova \& Preston, 2017). Understanding how we represent related information in memory in service of knowledge building and generalization has important implications beyond basic memory research. For example, in education, building complex knowledge is more important than remembering where one learned a single fact. Inversely, in eye-witness testimony, source specificity is critical and false alarms to any inferred information can be detrimental. Finally, understanding how information learned about one individual may be spontaneously generalized to others helps us understand how memory biases decisions. 


\section{Acknowledgments}

This work was supported in part by the National Institute for Neurological Disease and Stroke Grant R01-NS- 112366 awarded to Dagmar Zeithamova.

Data available through the Open Science Framework:

https://osf.io/3w87a/?view only=ca338682927a4af7af9e639e62354b68

The authors declare no competing financial interests. 


\section{References}

Ashby, S. R., Bowman, C. R., \& Zeithamova, D. (2020). Perceived similarity ratings predict generalization success after traditional category learning and a new paired-associate learning task. Psychonomic Bulletin \& Review, 27(4), 791-800. https://doi.org/10.3758/s13423-020-01754-3

Banino, A., Koster, R., Hassabis, D., \& Kumaran, D. (2016). Retrieval-Based Model Accounts for Striking Profile of Episodic Memory and Generalization. Scientific Reports, 6. https://doi.org/10.1038/srep31330

Barron, H. C., Reeve, H. M., Koolschijn, R. S., Perestenko, P. V., Shpektor, A., Nili, H., Rothaermel, R., Campo-Urriza, N., O’Reilly, J. X., Bannerman, D. M., Behrens, T. E. J., \& Dupret, D. (2020). Neuronal Computation Underlying Inferential Reasoning in Humans and Mice. Cell, 183(1), 228243.e21. https://doi.org/10.1016/j.cell.2020.08.035

Bowman, C. R., Iwashita, T., \& Zeithamova, D. (2020, November 26). Tracking prototype and exemplar representations in the brain across learning. ELife; eLife Sciences Publications Limited. https://doi.org/10.7554/eLife.59360

Bunsey, M., \& Eichenbaum, H. (1996). Conservation of hippocampal memory function in rats and humans. Nature, 379(6562), 255-257. https://doi.org/10.1038/379255a0

Cai, D. J., Aharoni, D., Shuman, T., Shobe, J., Biane, J., Song, W., Wei, B., Veshkini, M., La-Vu, M., Lou, J., Flores, S. E., Kim, I., Sano, Y., Zhou, M., Baumgaertel, K., Lavi, A., Kamata, M., Tuszynski, M., Mayford, M., ... 
Silva, A. J. (2016). A shared neural ensemble links distinct contextual memories encoded close in time. Nature, 534(7605), 115-118. https://doi.org/10.1038/nature17955

Carpenter, A. C., \& Schacter, D. L. (2017). Flexible retrieval: When true inferences produce false memories. Journal of Experimental Psychology: Learning, Memory and Cognition, 43(3), 335-349.

Carpenter, A. C., \& Schacter, D. L. (2018a). False memories, false preferences: Flexible retrieval mechanisms supporting successful inference bias novel decisions. Journal of Experimental Psychology: General, 147(7), 988-1004. https://doi.org/10.1037/xge0000391

Carpenter, A. C., \& Schacter, D. L. (2018b). Flexible retrieval mechanisms supporting successful inference produce false memories in younger but not older adults. Psychology and Aging, 33(1), 134-143. https://doi.org/10.1037/pag0000210

Chanales, A. J. H., Tremblay-McGaw, A. G., Drascher, M. L., \& Kuhl, B. A. (2021). Adaptive Repulsion of Long-Term Memory Representations Is Triggered by Event Similarity. Psychological Science, 32(5), 705-720. https://doi.org/10.1177/0956797620972490

Collie, A., Myers, C., Schnirman, G., Wood, S., \& Maruff, P. (2002). Selectively impaired associative learning in older people with cognitive decline. Journal of Cognitive Neuroscience, 14(3), 484-492. https://doi.org/10.1162/089892902317361994 
Diana, R. A., Yonelinas, A. P., \& Ranganath, C. (2007). Imaging recollection and familiarity in the medial temporal lobe: A three-component model. Trends in Cognitive Sciences, 11(9), 379-386. https://doi.org/10.1016/j.tics.2007.08.001

Doll, B. B., Shohamy, D., \& Daw, N. D. (2015). Multiple memory systems as substrates for multiple decision systems. Neurobiology of Learning and Memory, 117, 4-13. https://doi.org/10.1016/j.nlm.2014.04.014

Duncan, K., Sadanand, A., \& Davachi, L. (2012). Memory 's Penumbra: Episodic Memory Decisions Induce Lingering Mnemonic Biases. 337(July), 485-488.

Edwards, C. A., Jagielo, J. A., Zentall, T. R., \& Hogan, D. E. (1982). Acquired equivalence and distinctiveness in matching to sample by pigeons: Mediation by reinforcer-specific expectancies. Journal of Experimental Psychology: Animal Behavior Processes. https://doi.org/10.1037/00977403.8.3.244

Eichenbaum, H. (2001). The hippocampus and declarative memory: Cognitive mechanisms and neural codes. Behavioural Brain Research, 127(1-2), 199207. https://doi.org/10.1016/S0166-4328(01)00365-5

Foerde, K., \& Steinglass, J. E. (2017). Decreased Feedback Learning in Anorexia Nervosa Persists After Weight Restoration. International Journal of Eating Disorders, 50(4), 415-423. https://doi.org/10.1002/eat.22709.Decreased

Honey, R. C., \& Hall, G. (1991). Acquired Equivalence and Distinctiveness of Cues Using a Sensory-Preconditioning Procedure. The Quarterly Journal of 
Experimental Psychology Section B, 43(2), 121-135.

https://doi.org/10.1080/14640749108401263

Hulbert, J. C., \& Norman, K. A. (2015). Neural Differentiation Tracks Improved Recall of Competing Memories Following Interleaved Study and Retrieval Practice. Cerebral Cortex (New York, N.Y.: 1991), 25(10), 3994-4008. https://doi.org/10.1093/cercor/bhu284

Keppel, G., \& Underwood, B. J. (1962). Proactive inhibition in short-term retention of single items. Journal of Verbal Learning and Verbal Behavior, 1(3), 153161. https://doi.org/10.1016/S0022-5371(62)80023-1

Koster, R., Chadwick, M. J., Chen, Y., Berron, D., Banino, A., Düzel, E., Hassabis, D., \& Kumaran, D. (2018). Big-Loop Recurrence within the Hippocampal System Supports Integration of Information across Episodes. Neuron, 99(6), 1342-1354. https://doi.org/10.1016/j.neuron.2018.08.009

Kumaran, D. (2012). What representations and computations underpin the contribution of the hippocampus to generalization and inference? Frontiers in Human Neuroscience, 6.

https://www.frontiersin.org/articles/10.3389/fnhum.2012.00157

Kumaran, D., \& McClelland, J. L. (2012a). Generalization through the recurrent interaction of episodic memories: A model of the hippocampal system. Psychological Review, 119(3), 573-616. https://doi.org/10.1037/a0028681

Kumaran, D., \& McClelland, J. L. (2012b). Generalization through the recurrent interaction of episodic memories: A model of the hippocampal system. Psychological Review, 119(3), 573-616. https://doi.org/10.1037/a0028681 
Mack, M. L., Love, B. C., \& Preston, A. R. (2018). Building concepts one episode at a time: The hippocampus and concept formation. Neuroscience Letters, 680, 31-38. https://doi.org/10.1016/j.neulet.2017.07.061

Mack, M. L., Preston, A. R., \& Love, B. C. (2013). Decoding the Brain's Algorithm for Categorization from Its Neural Implementation. Current Biology, 23(20), 2023-2027. https://doi.org/10.1016/j.cub.2013.08.035

Marsh, E. J., Cantor, A. D., \& Brashier, N. M. (2016). Believing that humans swallow spiders in their sleep: False beliefs as side effects of the processes that support accurate knowledge. In The psychology of learning and motivation (pp. 93-132). Elsevier Academic Press.

Meeter, M., Shohamy, D., \& Myers, C. E. (2009). Acquired Equivalence Changes Stimulus Representations. Journal of the Experimental Analysis of Behavior, 91(2), 127-141. https://doi.org/10.1901/jeab.2009.91-127

Minda, J. P., \& Smith, J. D. (2011). Prototype models of categorization: Basic formulation, predictions, and limitations. In Formal approaches in categorization (pp. 40-64). Cambridge University Press. https://doi.org/10.1017/CBO9780511921322.003

Norman, K. A., Polyn, S. M., Detre, G. J., \& Haxby, J. V. (2006). Beyond mindreading: Multi-voxel pattern analysis of fMRI data. In Trends in Cognitive Sciences (Vol. 10, Issue 9, pp. 424-430). https://doi.org/10.1016/j.tics.2006.07.005 
Nosofsky, R. M. (1986). Attention, similarity, and the identification-categorization relationship. Journal of Experimental Psychology: General, 115(1), 39-57. https://doi.org/10.1037/0096-3445.115.1.39

Pan, X., Fan, H., Sawa, K., Tsuda, I., Tsukada, M., \& Sakagami, M. (2014). Reward Inference by Primate Prefrontal and Striatal Neurons. Journal of Neuroscience, 34(4), 1380-1396. https://doi.org/10.1523/JNEUROSCI.2263-13.2014

Polyn, S. M., Natu, V. S., Cohen, J. D., \& Norman, K. A. (2005). Category-specific cortical activity precedes retrieval during memory search. Science (New York, N.Y.), 310(5756), 1963-1966. https://doi.org/10.1126/science.1117645

Posner, M. I., \& Keele, S. W. (1968). On the genesis of abstract ideas. Journal of Experimental Psychology, 77(3, Pt.1), 353-363. https://doi.org/10.1037/h0025953

Preston, A. R., Shrager, Y., Dudukovic, N. M., \& Gabrieli, J. D. E. (2004). Hippocampal contribution to the novel use of relational information in declarative memory. Hippocampus, 14, 148-152.

Rashid, A. J., Yan, C., Mercaldo, V., Hsiang, H.-L. (Liz), Park, S., Cole, C. J., De Cristofaro, A., Yu, J., Ramakrishnan, C., Lee, S. Y., Deisseroth, K., Frankland, P. W., \& Josselyn, S. A. (2016). Competition between engrams influences fear memory formation and recall. Science, 353(6297), 383-387. https://doi.org/10.1126/science.aaf0594 
Richter, F. R., Chanales, A. J. H., \& Kuhl, B. A. (2016). Predicting the integration of overlapping memories by decoding mnemonic processing states during learning. Neurolmage, 124, 323-335.

https://doi.org/10.1016/j.neuroimage.2015.08.051

Schlichting, M. L., Mumford, J. A., \& Preston, A. R. (2015). Learning-related representational changes reveal dissociable integration and separation signatures in the hippocampus and prefrontal cortex. Nature Communications, 6, 8151. https://doi.org/10.1038/ncomms9151

Schlichting, M. L., \& Preston, A. R. (2017). The hippocampus and memory integration: Building knowledge to navigate future decisions. In M. C. Duff \& D. E. Hannula (Eds.), The Hippocampus from Cells to System: Structure, Connectivity, and Functional Contributions to Memory and Flexible Cognition (pp. 405-437). Springer.

Schlichting, M. L., Zeithamova, D., \& Preston, A. R. (2014). CA1 subfield contributions to memory integration and inference. Hippocampus, 24(10), 1248-1260. https://doi.org/10.1002/hipo.22310

Scoville, W. B., \& Milner, B. (1957). Loss of recent memory after bilateral hippocampal lesions. The Journal of Neuropsychiatry and Clinical Neurosciences, 20(11), 11-21. https://doi.org/10.1136/jnnp.20.1.11

Shohamy, D., \& Wagner, A. D. (2008). Integrating Memories in the Human Brain: Hippocampal-Midbrain Encoding of Overlapping Events. Neuron, 60(2), 378-389. https://doi.org/10.1016/j.neuron.2008.09.023 
Squire, L. R. (1992). Memory and the hippocampus: A synthesis from findings with rats, monkeys, and humans. Psychological Review, 99(2), 195-231. https://doi.org/10.1037/0033-295X.99.3.582

Taylor, J. E., Cortese, A., Barron, H. C., Pan, X., Sakagami, M., \& Zeithamova, D. (2021). How do we generalize? (arXiv:2104.00899). arXiv. http://arxiv.org/abs/2104.00899

Tse, D., Langston, R. F., Kakeyama, M., Bethus, I., Spooner, P. A., Wood, E. R., Witter, M. P., \& Morris, R. G. M. (2007). Schemas and memory consolidation. Science (New York, N.Y.), 316(5821), 76-82. https://doi.org/10.1126/science.1135935

Underwood, B. J. (1969). Attributes of memory. Psychological Review, 76(6), 559. https://doi.org/10.1037/h0028143

Varga, N. L., Gaugler, T., \& Talarico, J. (2019). Are mnemonic failures and benefits two sides of the same coin?: Investigating the real-world consequences of individual differences in memory integration. Memory \& Cognition, 47(3), 496-510. https://doi.org/10.3758/s13421-018-0887-4

Zalesak, M., \& Heckers, S. (2009). The role of the hippocampus in transitive inference. Psychiatry Research: Neuroimaging, 172(1), 24-30. https://doi.org/10.1016/j.pscychresns.2008.09.008

Zeithamova, D., \& Bowman, C. R. (2020). Generalization and the hippocampus: More than one story? Neurobiology of Learning and Memory, 175, 107317. https://doi.org/10.1016/j.nlm.2020.107317 
Zeithamova, D., Dominick, A. L. L., \& Preston, A. R. R. (2012). Hippocampal and ventral medial prefrontal activation during retrieval-mediated learning supports novel inference. Neuron, 75(1), 168-179.

https://doi.org/10.1016/j.neuron.2012.05.010

Zeithamova, D., \& Preston, A. R. (2010). Flexible memories: Differential roles for medial temporal lobe and prefrontal cortex in cross-episode binding. The Journal of Neuroscience: The Official Journal of the Society for Neuroscience, 30(44), 14676-14684.

https://doi.org/10.1523/JNEUROSCI.3250-10.2010

Zeithamova, D., \& Preston, A. R. (2017). Temporal proximity promotes integration of overlapping events. Journal of Cognitive Neuroscience, 29(8), 13111323. https://doi.org/10.1162/jocn_a_01116

Zeithamova, D., Schlichting, M. L., \& Preston, A. R. (2012). The hippocampus and inferential reasoning: Building memories to navigate future decisions. Frontiers in Human Neuroscience, 6(March), 1-14. https://doi.org/10.3389/fnhum.2012.00070 